Les Cahiers du Gres

\section{Des nouvelles du GRES}

Volume 5, numéro 1, printemps 2005

URI : https://id.erudit.org/iderudit/010882ar

DOI : https://doi.org/10.7202/010882ar

Aller au sommaire du numéro

\section{Éditeur(s)}

Groupe de Recherche Ethnicité et Société

CEETUM

ISSN

1499-0431 (imprimé)

1499-044X (numérique)

Découvrir la revue

Citer ce document

(2005). Des nouvelles du GRES. Les Cahiers du Gres, 5(1), 101-104.

https://doi.org/10.7202/010882ar
Les Cahiers du Gres Série Diversité urbaine Groupe de Recherche Ethnicte et Societs 


\section{Des nouvelles du GRES}

Comité de lecture : un nouveau collaborateur à l'étranger: Christian Rinaudo.

Nous avons le plaisir d'accueillir un nouveau correspondant aux Cahiers, à titre de collaborateur à l'étranger. Sociologue, il est maître de Conférences à l'Université de Nice-Sophia Antipolis et chercheur à l'unité de recherche Migrations et Sociétés SOLIIS-URMIS (CNRS - Université Denis Diderot, Paris VII - Université de Nice - Sophia Antipolis). Ses recherches portant, entre autres, sur la construction sociale de l'ethnicité en milieu urbain et sur le traitement $\mathrm{du}$ racisme et des discriminations dans le monde du travail seront d'un apport certain. Mentionnons un important ouvrage qu'il a publié en 1999 dans lequel il traitait de la construction sociale de l'ethnicité : L'ethnicité dans la cité, Jeux et enjeux de la catégorisation ethnique. Éditions L'Harmattan, Paris.

\section{De nouveaux projets de recherche}

L'année 2004 a été riche en nouveaux projets de recherche abordant la diversité en milieu montréalais. Mentionnons :

ForTIN, Sylvie. "Pratiques cliniques et diversité urbaine: les défis d'une pédiatrie en milieu hospitalier montréalais ". Fonds de la recherche en santé du Québec (FRSQ), programme de chercheur boursier, 2004-2008 et subvention de recherche 2004-2007.
ForTin, Sylvie, Fernando Alvarez, Gilles Bibeau et Danielle Laudy. "Pratiques cliniques hospitalières et pluralisme urbain: L'arrimage d'une pédiatrie à la diversité de son milieu ». Institut de recherche en santé du Canada (IRSC), 2004-2007.

Fortin, Sylvie, Marie-Nathalie LeBlanc et Josiane Le Gall. "Stratégies identitaires et pratiques sociales des musulmans francophones de Montréal ", Conseil de recherches en sciences humaines du Canada (CRSH), 2004-2007.

Isin, Engin; Daiva Stasiulis, Danielle Juteau et Janine Brodie."Recasting Social Citizenship». Conseil de recherche en sciences humaines du Canada (CRSH) - Subventions pour la conception de réseaux stratégiques de recherches, 2004-2005.

LeBlanc, Marie-Nathalie; Deirdre Meintel, Patricia Lamarre et Josiane LE GaLl. "Solidarity and Community Among Youth: The Case of Montreal's Pluriethnic Hip-Hop Culture ». Conseil de recherches en sciences humaines du Canada (CRSH), 2004-2007.

Le Gall, Josiane; Catherine Montgomery, Hélène Belleau, Deirdre MeinTel et Alex Battaglini. "Trajectoires et déterminants de l'utilisation des services sociaux et de santé : perspectives des hommes récemment immigrés ", Fonds québécois de la recherche sur la société et la culture (FQRSC), 20042006. 
LE Gall, Josiane; Hélène Belleau, Deirdre Meintel et MarieNathalie LeBlanc. "L'entrée dans la vie amoureuse des filles et garçons de diverses origines ethniques ". Projet pilote en cours, CLSC Nord de l'Ile, Montréal, 2003-2004.

MeInTEL, Deirdre. "Trajectoires des spiritualistes à Montréal ». Conseil de recherches en sciences humaines du Canada (CRSH) petite subvention, 2004.

Renaud, Jean, Danielle Juteau, Deirdre Meintel, Marie-Nathalie LeBlanc, Victor Piché et 19 autres chercheurs. Centre d'études ethniques des universités montréalaises. Regroupement stratégique, centre de recherche. Fonds québécois de la recherche sur la société et la culture (FQRSC), 20042010.

Renaud, Jean, Victor Piché . " Les mécanismes d'insertion aux communautés francophone et anglophone ». Fonds québécois de la recherche sur la société et la culture (FQRSC), 2003-2005.

St-Cyr, Denise; Hélène Belleau, Sylvie Gendron et Josiane Le Gall, « Le transfert des connaissances entre praticiens, gestionnaires et chercheurs comme source d'innovation en CLSC ». Fonds québécois de la recherche sur la société et la culture (FQRSC), 20042006.

Young, Robert; Danielle JuTEAu et 64 autres chercheurs. "Multilevel Governance and Public Policy in Canadian Municipalities.» Conseil de recherche en sciences humaines du Canada (CRSH) - Grands travaux de recherche concertée, 2004-2009.

\section{Stagiaires}

Nous avons été très heureux d'accueillir en 2004-2005 plusieurs stagiaires, du Québec et de l'extérieur, qui ont contribué au dynamisme du GRES et du CEETUM.

Susana Moreno Maestro, étudiante de doctorat en anthropologie à l'Universidad de Sevilla et boursière du Conseil d'Éducation et de Sciences de la Réunion de l'Andalousie. À travers son stage, elle vise à comparer les politiques d'immigration au Canada et au Québec, en relation à la question nationale. Ce projet permettra d'enrichir sa thèse qui porte sur l'analyse des perceptions, des formes de gestion, des stratégies politiques et discours en ce qui concerne la situation multiculturelle existante en Andalousie.

Sonia Das, doctorante en anthropologie de l'University of Michigan et boursière du « funding to the Fulbright IEE Organization » (Institute of International Education) des États-Unis. Elle travaille sur les Tamouls à Montréal en milieu scolaire et s'intéresse aux questions relatives aux langues. Son projet de recherche s'intitule "Who is Québécois? The Articulation of Nationalist and Multicultural Interests in Emerging Language Ideologies. ».

Mélanie Le Blanc, boursière du CRSH au doctorat en sciences du langage de l'Université de Moncton au Nouveau-Brunswick. Elle a poursuivi à Montréal son projet qui porte sur la sociolinguistique, les représentations et les idéologies linguistiques, elle participe également très activement au projet de recherche "Prise de Parole II, la francophonie canadienne et la nouvelle économie mondialisée » (CRSH). 
Karolina Micor, étudiante en DESS français langue étrangère de l'Université d'Avignon en France dont le projet de recherche porte sur «Les immigrants polonais de la deuxième génération, nés à Montréal et parlant au moins trois langues ».

En outre, Robbyn Seller, anthropologue, à titre de stagiaire postdoctorale poursuit ses recherches sur les migrants des Caraïbes à Montréal et Amantina Osorio, doctorante en anthropologie poursuite sa thèse sur "Rapports familiaux et insertion sociale en région chez les ressortissants de l'ex-Yougoslavie».

Plusieurs membres du GRES collaborent activement sur des projets de recherche, dont le nouveau projet "Pratiques sociales et rituelles, les musulmans de Montréal » (CRSH 2004-2007), «Transmission culturelle et identitaire chez les jeunes couples mixtes » (FQRSC 2003-2006), ainsi que le projet «Prise de Parole II, la francophonie canadienne et la nouvelle économie mondialisée " (CRSH 20012004) dirigé par Monica Heller (Toronto).

Les assistants de recherche qui ont participé à ces projets incluent : Emmanuel Kahn, Marie-Jeanne Blain, Stéphanie Lamarre, Isabel Heck, Géraldine Mossière, Christelle Cassan, Caroline Désilets, Marie-Noëlle Fortin, Sandra Najac, Marie-Claude Bourbon, ainsi qu'Anne Catherine Kennedy, Amantina Osorio, Eric Meulan, Shamelkan Ghamraoui et Diahara Traore.

\section{Série Diversité Urbaine}

Les Cahiers sont maintenant en ligne sur la plateforme de diffusion www.Erudit.org. Ceci marque un nouveau pas vers une diffusion accrue de nos Cahiers, qui permettra d'assurer une plus grande la visibilité des travaux actuels et de favoriser les publications de chercheurs juniors.

\section{Quelques contributions, articles,} chapitres de livre ou monographie :

Armand, F., Dagenais, D. et Lamarre, P. (à paraître) " Programmes d'immersion et élèves plurilingues au Canada.»Actes de colloque CEFISEM $d u$ rectorat de l'académie d'Amiens, Amiens, 18-19 avril.

Belleau, Hélène et LE GALl, Josiane, 2004. "Les jeunes d'ici et d'ailleurs : de la rencontre des valeurs à la distinction des genres ", dans Pronovost, G. et Royer, C. (dir.), Les valeurs des jeunes Sainte-Foy, PUQ, p. 188-204.

Fortin, Sylvie, 2004. «Issues and Challenges of Pediatrics in Pluriethnic Settings ", Ethnicity \& Health, vol. 9, $\mathrm{n}^{\circ} 1$, p. 43.

Fortin, Sylvie, 2004. "Les enjeux et les défis d'une pratique pédiatrique en contexte pluraliste : réflexions théoriques ", dans S. De Plaen (dir.), Soins aux enfants et pluralisme culturel. Collection Intervenir, Hôpital SainteJustine, p. 87-105.

Fortin, Sylvie et Jean Renaud, 2004. "Stratégies d'établissement en c o n texte montréalais : une diversité de modalités?", dans J. Renaud, A.Germain et X. Leloup (éd.), Le racisme et la discrimination: les tensions 
et les défis d'une société plurielle. Les Presses de l'Université Laval, p. 3159.

Henry S., V. Piché, D. Ouédraogo, E.F. Lambin, 2004. "Descriptive analysis of the individual migratory pathways according to environmental ypologies ", Population and Environment, vol. 24, n ${ }^{\circ}$ 5, p. 397-422.

Juteau, Danielle, 2004. "Pure laine" Québécois in Quebec ", dans E. Kaufmann (dir.), Rethinking Ethnicity. Majority Groups and Dominant Minorities, Routledge, p. 84-101.

Lalou, R. et Victor Piché, 2004. "Les migrants face au SIDA: entre gestion des risques et contrôle social. L'exemple de la Vallée du Fleuve Sénégal ", Population, revue de l'INED, vol. 59, $\mathrm{n}^{\circ} 2$, mars-avril 2004, p. 233-268.

LEBLANC, Marie-Nathalie (à paraître). «De la tradition à l'Islam: L'orthodoxie à l'encontre des rites culturels", Cahiers des Etudes Africaines.

LeBlanc, Marie-Nathalie, 2005. «Identités islamiques et mouvance transnationale: les nouveaux rôles du Hajj chez les jeunes musulmans de Côte d'Ivoire dans les années 90 ", dans Fatou Sow and Muriel Perez-Gomez, L'islam politique en Afrique subsaharienne d'bier à aujourd'bui : discours, trajectoires et réseaux. Paris: Karthala.

Le Gall, Josiane et Deirdre MeIntel (sous presse). "Pratiques de nomination des enfants dans les unions mixtes au Québec : revendication d'une appartenance multiple ", dans F..R. Ouellete et A. Fine (dir.). L'octroi des noms. SteFoy : Presses de l'Université Laval.
Meintel, Deirdre et Emmanuel KAHN, (à paraître 2005). "Parents de la deuxième génération et projets de transmission identitaire ", dans M. Potvin, P. Eid et N. Venel (dir.), Les "deuxièmes générations» au Québec et en France : perspectives théoriques et empiriques. Paris, PUF.

Rally, Jean-Louis, Victor Piché et Patrick Simon (sous presse). "Démographie et ethnicité : une relation ambiguë », dans G. Caselli, J. Vallin et G. Wunsch(éds), Démographie: analyse et synthèse. Volume 6 : Conséquences des changements démographiques, Paris, INED.

Jean Renaud, Gustave Goldmann (2005, sous presse). "Les répercussions du 11 septembre 2001 sur l'établissement économique des nouveaux immigrants au Canada et au Québec ", Recherches sociographiques.

Renaud, Jean (2005). « Nouveaux immigrants au Québec. Du français minoritaire au français dominant ». In Virginie Conti et Jean-François de Pietro, L'intégration des migrants en terre francophone. Aspects linguistiques et sociaux. Le Mont-sur-Lausanne, Éditions LEP Loisirs et Pédagogie, p. 27-40. 